\title{
Imagens multiespectrais para avaliação de índice de área foliar e massa seca do capim 'Tifton 85', sob adubação nitrogenada
}

\author{
Multispectral imaging for leaf area index and dry mass evaluation of 'Tifton 85' bermudagrass \\ under nitrogen fertilization
}

\author{
Cláudia Rossini Simões ${ }^{\mathrm{I}}$ Roberto Oscar Pereyra Rossiello ${ }^{\mathrm{II}}$ Mariana Guimarães Graciosa ${ }^{\mathrm{I}}$ \\ Marley Lamounier Machado ${ }^{\text {III }}$ Camila Figueiredo da Silva ${ }^{\text {IV }}$
}

\section{RESUMO}

$O$ presente trabalho teve por objetivo analisar as respostas do capim 'Tifton 85' (Cynodon spp.) à adubação nitrogenada, em termos de área foliar verde e de massa seca da parte aérea, utilizando índices calculados a partir de informações contidas em imagens multiespectrais, em comparação com indicadores obtidos por métodos diretos. Os tratamentos consistiram de quatro doses de N-ureia $(0 ; 100 ; 150$ e $200 \mathrm{~kg}$ $\left.\mathrm{ha}^{-1}\right)$ dispostos em um desenho experimental inteiramente casualizado, com quatro repetições. Aos 26 dias, após um corte de uniformização, foram determinados os seguintes atributos do dossel: o Índice de Área Foliar (IAF), a massa seca da parte aérea (MSPA), o indice de cobertura verde (ICV), utilizando uma câmara digital na faixa do visível e o Índice de Vegetação por Diferença Normalizada (NDVI), utilizando uma câmara multiespectral. Os quatro atributos do dossel vegetativo responderam positivamente à adubação nitrogenada. Enquanto o ICV mostrou relações curvilineares com o IAF e a MSPA $\left(R^{2} I A F=0,89 ; R^{2} M S P A=0,87\right)$ o NDVI mostrou relações lineares $\left(r^{2} I A F=0,62 ; r^{2} M S P A=0,64\right)$, sem evidenciar tendência à saturação. Foi concluído que é possivel avaliar a adubação nitrogenada em pastagens de Cynodon spp., utilizando análise de imagens, e que tanto o NDVI quanto o ICV podem ser utilizados como indicadores de produtividade do capim Tifton 85.

Palavras-chave: Cynodon spp., indice de cobertura verde, indice de área foliar, NDVI, refletância do dossel.

\section{ABSTRACT}

The purpose of this study was to verify the responses of 'Tifton 85 ' bermudagrass (Cynodon spp.) to nitrogen fertilization in terms of green leaf area and aerial dry mass, using indices calculated from spectral information contained in multispectral images, in comparison with indicators obtained by direct methods. The bermudagrass was managed under four $N$ treatments $(0 ; 100$; 150 and $200 \mathrm{~kg} \mathrm{ha}^{-1}$ ) using a completely randomized experimental design, with four repetitions. Twenty six days after a standardization cut, four canopy traits were determined: leaf area index (LAI), aerial dry mass (ADM), green coverage index (GCI), using a $R G B$ camera and the Normalized Difference Vegetation Index (NDVI), using a multispectral camera. All the traits responded positively to nitrogen fertilization. However, while the GCI showed curvilinear relationships with LAI $\left(R^{2}=0.89\right)$ and ADM $\left(R^{2}=0.87\right)$, the NDVI showed linear relationships with both $\left(r^{2} L A I=0.62 ; r^{2} A D M=0.64\right)$ without evidences of saturated responses. It was concluded that it is possible to evaluate nitrogen fertilizer on pastures of Cynodon spp., using image analysis. NDVI and ICV can be used as productivity indicators of 'Tifton 85 ' bermudagrass.

Key words: canopy reflectance, Cynodon spp., Green Coverage Index, leaf area index, NDVI.

\section{INTRODUÇÃO}

Nos últimos anos, têm ganhado destaque os estudos sobre parametrização de atributos morfofisiológicos dos dosséis vegetais, feitos com métodos baseados nas propriedades ópticas das plantas (OLLINGER, 2011). Tais estudos envolvem o uso de técnicas radiométricas, que utilizam bandas espectrais específicas, ou sistemas baseados no processamento de imagens digitais obtidas por câmeras fotográficas (SENA JÚNIOR et al., 2008). Diversos índices gerados pela combinação de valores

\footnotetext{
IPrograma de Pós-graduação, Iinsttituto de Zotecnia (IZ), Universidade Federal Rural do Rio de Janeiro (UFRRJ), Seropédica, RJ, Brasil. IIDepartamento de Solos, Instituto de Agronomia, UFRRJ, Rod. BR 465, KM 7, 23890-000, Seropédica, RJ, Brasil. E-mail: rossielo@terra.com.br. Autor para correspondência.

IIIPrograma de Pós-graduação, Escola de Agronomia (EA), Universidade Federal de Viçosa (UFV), Viçosa, MG, Brasil.

${ }^{\text {IV }}$ Curso de Agronomia, UFRRJ, Seropédica, RJ, Brasil.
} 
de refletância entre bandas espectrais específicas são utilizados como indicadores da quantidade de vegetação e outros atributos dos dosséis, sendo o Índice de Vegetação por Diferença Normalizada (NDVI, do inglês Normalized Difference Vegetation Index), o mais conhecido (OLLINGER, 2011). Em relação às câmaras digitais, embora com menor resolução espectral em relação aos radiômetros (SENA JÚNIOR et al., 2008), o seu uso tem aumentado nos últimos anos, dada sua acessibilidade e facilidades operativas, com um mínimo de treinamento do usuário. $\mathrm{O}$ processamento analítico das imagens captadas com as câmeras permite a construção de diversos índices, relacionados com a cobertura vegetal, o acúmulo de biomassa, e os teores de nitrogênio em diversas espécies cultivadas (SENA JÚNIOR et al. 2008; LI et al., 2010). Todavia, no caso das espécies forrageiras, a validação dessas técnicas, dependerá das características estruturais e botânicas da vegetação em foco, o que implica no conhecimento da variabilidade de atributos como grau de cobertura, índice de área foliar (IAF) e acúmulo de biomassa dos dosséis (SCHUT \& KETELAARS, 2003; ROTZ et al., 2008).

O conhecimento da quantidade e qualidade da forragem disponível é essencial para a gestão da produção animal em pastagens (ZHAO et al., 2007). O acesso a essas informações tem sido feito tradicionalmente por corte e secagem da biomassa, seguida de análises laboratoriais (MANNETJE, 2000). Tais procedimentos, embora precisos, possuem limitações, relativas a custos e tempo de execução, o que destaca a importância das metodologias indiretas, que permitem efetuar tais avaliações em tempo real, a exemplo da utilização de informações espectrais obtidas de câmeras digitais e multiespectrais.

O gênero Cynodon é constituído por espécies e cultivares de ampla adaptação às condições próprias de ambientes tropicais e subtropicais (VILELA et al., 2005). Entre os diversos híbridos oriundos de cruzamentos intra ou interespecíficos, destaca-se a cultivar 'Tifton 85' (Cynodon spp.), por combinar elevado potencial de produção de forragem com alta digestibilidade (QUARESMA et al., 2011). O nitrogênio (N) é essencial à expressão da produtividade do capim 'Tifton 85 ', tanto por efeitos diretos do nutriente sobre o IAF e a consequente interceptação da radiação solar (OLIVEIRA et al., 2010), quanto por efeitos indiretos, ligados à absorção e assimilação fotossintética do carbono (ALDERMAN et al., 2011; SILVA et al., 2011).

Dadas as possibilidades oferecidas pelas técnicas de análise de imagens provenientes de câmeras digitais, a hipótese deste trabalho foi a de que é possível avaliar os efeitos da adubação nitrogenada no capim 'Tifton 85' através dessas ferramentas. Nesse sentido, os objetivos foram: a) verificar o comportamento de dois índices calculados a partir de imagens digitais, a saber: o Índice de Cobertura Verde (ICV), obtido com câmera fotográfica comum e o NDVI, obtido com câmera multiespectral, em relação à adubação nitrogenada; b) determinar se tais índices podem servir como indicadores de produtividade do capim 'Tifton 85 '.

\section{MATERIAL E MÉTODOS}

O experimento foi conduzido na Estação Experimental de Parasitologia "Wilhelm Otto Neitz" do Instituto de Veterinária da UFRRJ, Seropédica,

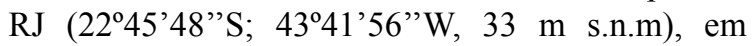
área de ocorrência de Planossolo Háplico, textura superficial arenosa (EMBRAPA, 2006). O plantio do capim 'Tifton 85 ' foi realizado em 2006, conforme os procedimentos detalhados por Oliveira et al. (2010). As unidades experimentais são constituídas por parcelas de $16 \mathrm{~m}^{2}$, sendo a parte central (2 $\left.\mathrm{m} \mathrm{x} 2 \mathrm{~m}\right)$ considerada a área útil para finalidade de amostragem. No mês de dezembro de 2011 foi realizado um corte de uniformização, a uma altura de $0,05 \mathrm{~m}$ do solo, seguido de uma aplicação basal de cloreto de potássio equivalente a $160 \mathrm{~kg} \mathrm{~K}_{2} \mathrm{O} \mathrm{ha}^{-1}$, em todas as unidades experimentais.

O experimento foi desenvolvido entre os meses de janeiro e março de 2012. O delineamento foi inteiramente casualizado, com quatro repetições. Os tratamentos consistiram em quatro doses de $\mathrm{N}(0$; $50 ; 100$ e $200 \mathrm{~kg} \mathrm{ha}^{-1}$ ) na forma de sulfato de amônio, aplicadas no início do mês de fevereiro, após um corte de uniformização. Em 29 de fevereiro de 2012, aos 26 dias após o corte (26 DAC), foram obtidas as imagens digitais necessárias à determinação dos índices de vegetação estudados, em conjunto com as amostragens de biomassa e IAF. Para a determinação do índice de Cobertura Verde (ICV), foram adquiridas imagens com uma câmera digital (CD) marca Sony, modelo DSC-W50, entre as 7h30min e 9h horas. Um suporte de PVC, em formato de "L", foi feito para o acoplamento ortogonal da câmera, a uma altura padrão de 1,60 m. As imagens obtidas (duas por parcela) foram transferidas para um computador e armazenadas no formato TIFF. Subsequentemente foram coletadas amostras da biomassa em pé, dentro de um quadrante com $0,04 \mathrm{~m}^{2}$, por corte rente ao solo, as quais foram mantidas em caixa isotérmica, à sombra. A coleta de imagens para a estimativa da refletância do dossel foi realizada entre as 11 e 13 
horas, sob condições de céu claro. Utilizou-se uma câmera multiespectral (CME) marca FluxData, modelo FD-1665-MS 5 (Channel Camera, Flux Data, Inc., NY), configurada nas seguintes bandas: azul (400-510nm); verde (500-580nm); vermelho (585$640 \mathrm{~nm})$, "red-edge" (720-730nm) e infravermelho próximo (770- $830 \mathrm{~nm})$. A CME foi posicionada a uma altura de 3,0 m e direcionada a nadir, utilizando uma estrutura metálica, sendo conectada a um computador, para permitir a visualização das imagens no momento da sua obtenção. Em cada parcela, foram tomadas três imagens, com resolução espacial de $0,33 \mathrm{~mm} /$ pixel. No campo de visão da imagem, era posicionado um painel padrão Spectralon (Labsphere, Inc., North Sutton, NH, USA), contendo quatro tons de cinza: branco difuso, cinza claro, cinza médio e cinza escuro, com refletâncias de 99\%, 75\%, 40\% e $10 \%$, respectivamente, para permitir a conversão dos valores de pixel em valores de refletância. $\mathrm{O}$ nível de refletância do dossel, dentro da faixa 400-700 nm, foi também avaliado com dois sensores de quantum (LI-190) conectados a radiômetros portáteis (marca LI-COR, modelo LI-250A) (LI-COR, Inc., Lincoln, $\mathrm{NE}$ ), posicionados a uma altura de $0,5 \mathrm{~m}$ acima da superfície do relvado e apontados para cima e para abaixo, consecutivamente.

As imagens obtidas com CD foram processadas e analisadas com auxílio do programa MATLAB v. 9 (MATHWORKS, 2000). Na etapa de pré-processamento, foram escolhidos pontos representativos de pixels de vegetação verde ou de solo nu ou com vegetação seca, para criar um arquivo com o vetor classificador, baseado na função discriminante de Fisher (JOHNSON \& WICHERN, 1999). Este procedimento permitiu a binarização da imagem em branco e preto, em que os pontos pretos representaram a vegetação verde. As imagens foram, então, recortadas de '2816C x 2112L' para '2040C x 2040L', correspondente a um campo de visão de $1 \mathrm{~m}^{2}$ e resolução espacial de $0,49 \mathrm{~mm} /$ pixel, e processadas para a obtenção do ICV, que corresponde ao porcentual de pixels verdes em relação ao número total de pixels da imagem. As imagens de pixels obtidas com CME foram convertidas em imagens de refletância, baseadas em cinco equações de calibração, uma para cada banda espectral, resultantes de regressão linear simples, entre os valores de pixels das imagens referentes a cada tom de cinza do painel Spectralon e os respectivos valores de refletância fornecidos pelo fabricante, de acordo com a equação (1): $\hat{\mathrm{Y}}_{\mathrm{ijk}}=\mathrm{a}+\mathrm{b} \mathrm{P}_{\mathrm{ijk}}$

em que: $\quad \mathrm{P}_{\mathrm{ijk}}=$ valor do pixel na linha $i$ e coluna $j$ da banda $k$, adimensional; $\hat{\mathrm{Y}}_{i j k}=$ refletância na linha $i$ e coluna $j$ da banda $k$, em $\%$; a e b $=$ coeficientes de regressão. Procedimento similar foi descrito por Abrahão et al. (2013). O processamento das imagens foi realizado com auxílio do programa MATLAB v. 9 (MATHWORKS, 2000). No presente estudo, foi utilizado o NDVI, definido como (2):

$\mathrm{NDVI}=(\mathrm{IVP}-\mathrm{V}) /(\mathrm{IPV}+\mathrm{V})$

em que: IPV $=$ valor de refletância na banda do infravermelho próximo; $\mathrm{V}=$ valor de refletância na banda do vermelho.

A biomassa colhida no campo foi imediatamente fracionada no laboratório, separandose o material verde do material morto ou senescente. O material verde foi separado em lâminas foliares e colmo+bainhas. Sub-amostras, contendo de 10 a $20 \%$ da massa fresca de lâminas foliares, foram separadas e digitalizadas diretamente em scanner, com uma resolução de 200 dpi. As imagens obtidas foram arquivadas no formato JPEG e analisadas com auxílio do software SIARCS ${ }^{\circledR}$, para a determinação da sua área superficial. Subsequentemente, o material foi posto a secar em estufa com circulação forçada de ar $\left(65^{\circ} \mathrm{C}\right)$, por $48 \mathrm{~h}$. Após pesagem, os dados foram utilizados para computar a massa seca de lâminas foliares (MSLF, $\mathrm{kg}$ $\mathrm{m}^{-2}$ ), a área foliar específica (AFE, $\mathrm{m}^{2}$ folha $\mathrm{kg} \mathrm{MS}$ folha ${ }^{-1}$ ), o IAF (calculado como AFE x MSLF) e a massa seca da parte aérea (MSPA, $\mathrm{kg} \mathrm{m}^{-2}$ ) do capim.

Os dados meteorológicos relativos ao período experimental foram obtidos junto ao Posto da Estação Experimental de Seropédica, da PESAGRORJ. Durante o mês de fevereiro de 2012, a precipitação pluviométrica total foi de $42,6 \mathrm{~mm}$, ou seja, um terço da normal climática regional (MATTOS et al., 1998). Houve elevação das temperaturas médias do ar, com mínima de $21,1^{\circ}$ e máxima de $31,3^{\circ}$. Esse quadro ambiental prejudicou a velocidade de rebrota do capim, sendo atenuado por regas, durante os dias de maior demanda evapotranspirativa.

Os dados foram submetidos à análise estatística para verificar a significância do fator dose de $\mathrm{N}$ (teste $\mathrm{F}, \mathrm{P} \leq 0,05$ ). Em atenção à natureza quantitativa da variável independente, usaram-se técnicas de regressão para a parametrização das respostas à adubação nitrogenada. Da mesma forma, em consonância com os objetivos deste estudo, as interrelações entre as variáveis experimentais, medidas ou estimadas, foram analisadas por regressão, com auxílio do software GraphPad Prism v. 5.0. Quando foi necessário optar entre modelos de regressão, utilizou-se o Critério de Informação de Akaike, já incluído no software, para a escolha do modelo mais provável. 


\section{RESULTADOS E DISCUSSÃO}

Aos 26 DAC, os dosséis já tinham acumulado uma quantidade apreciável de cobertura verde, de forma que os níveis de radiação fotossinteticamente ativa interceptada $(400-700 \mathrm{~nm})$ oscilaram entre $35,8 \pm 3,2$ (testemunha) e 71,1 $\pm 2,8$ $\%\left(200 \mathrm{~kg} \mathrm{~N} \mathrm{ha}^{-1}\right)$. Esses valores foram menores que os observados na mesma área, em anos anteriores (OLIVEIRA et al., 2010), possivelmente em razão da baixa precipitação pluviométrica no ciclo de rebrota estudado. As variáveis estudadas (IAF, ICV, MSPA e NDVI) responderam positivamente à adubação nitrogenada. Tanto o IAF (Figura 1A) quanto o ICV (Figura 1B), guardaram uma relação linear com as doses de $\mathrm{N}$ aplicadas. $\mathrm{O}$ alto grau de associação entre essas variáveis deveu-se, em parte, à própria metodologia de determinação, a qual excluiu a área foliar senescente ou morta, manualmente, no caso do IAF, ou através da aplicação de algoritmos específicos, no caso da classificação das imagens.

Os dados coletados com os sensores quânticos indicaram que a refletância média do dossel, dentro da faixa fotossinteticamente ativa (400-700nm), decresceu significativamente $(\mathrm{P}<0,05)$, de 8,4 para 5,3\%, entre as doses de zero e $200 \mathrm{~kg} \mathrm{~N}$ há $^{-1}$, respectivamente. A maior absorção de RFA pela clorofila $a$ está associada aos comprimentos de onda na faixa do vermelho (ABRAHÃO et al. , 2013), justificando as reduções da refletância observadas dentro da faixa visível do espectro (XIONG et al., 2007). Por outro lado, a refletância na banda do infravermelho aumenta de forma contínua com os aumentos em área foliar ou MSPA (OLLINGER, 2011). A consequência desse comportamento espectral da vegetação é o aumento do NDVI com a

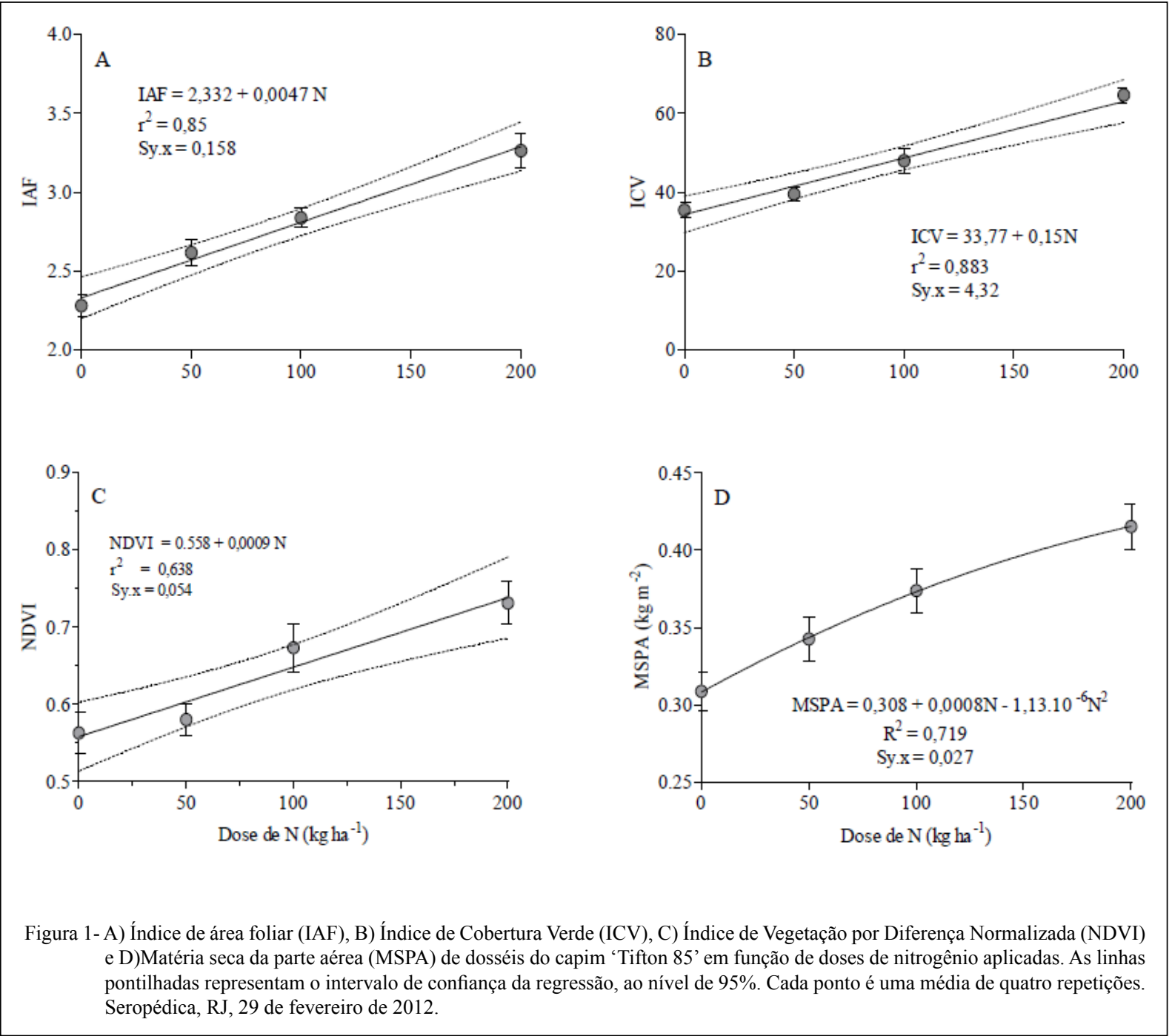

Ciência Rural, v.45, n.4, abr, 2015. 
dose de $\mathrm{N}$ aplicada (Figura 1C). Todavia, o modelo linear obtido entre NDVI e dose de $\mathrm{N}$ teve um coeficiente de determinação menor $\left(\mathrm{r}^{2}=0,63\right)$ que os correspondentes ao IAF e ao ICV (Figura 1A e B), evidenciando um aumento da variabilidade dentro de tratamentos. Parte dessa variabilidade pode ser atribuída a flutuações nos teores de $\mathrm{N}$ disponível, entre repetições, as quais afetam componentes importantes da geometria do dossel no capim 'Tifton 85', como altura e distribuição angular de folhas e estolões (ROSSIELLO \& ANTUNES, 2012). Em relação à resposta da produção da MSPA à adubação nitrogenada, ela evidenciou um padrão de resposta quadrático (Figura 1D), similar ao observado em experimentos anteriores (SILVA et al., 2011).

Foi verificado que o ICV relacionouse de forma curvilinear tanto com o IAF (Figura 2A) quanto com a MSPA (Figura 2B). No caso da relação com o IAF, o modelo de melhor ajuste foi o sigmoidal, que indicou, para valores de IAF
$>3,0$, uma tendência crescente à estabilização do ICV (Figura 2A). Isso significa que incrementos adicionais de IAF implicarão em incrementos progressivamente menores do ICV. A implicação é que um atributo como o ICV deva guardar uma relação assintótica com o IAF, tal como acontece com a fração da RFA incidente interceptada pelo dossel (OLIVEIRA et al., 2010). Já com relação à variação de MSPA induzida pela adubação nitrogenada, o ICV relacionou-se de forma exponencial (Figura 2B) sem evidenciar tendência à saturação, em função de valores de biomassa não excessivamente elevados. $\mathrm{Na}$ figura 2, também são apresentadas as relações entre o NDVI e o IAF (Figura 2C) ou a MSPA (Figura 2D), ambas de natureza linear, mas com coeficientes de determinação menores $\left(0,62<\mathrm{r}^{2}<0,65\right)$ do que os indicados na figura $2 \mathrm{~A}$ e $2 \mathrm{~B}$. Esse aumento na variabilidade experimental pode ser consequência de que as estimativas de refletância foram baseadas na estrutura intacta do dossel, enquanto as de área

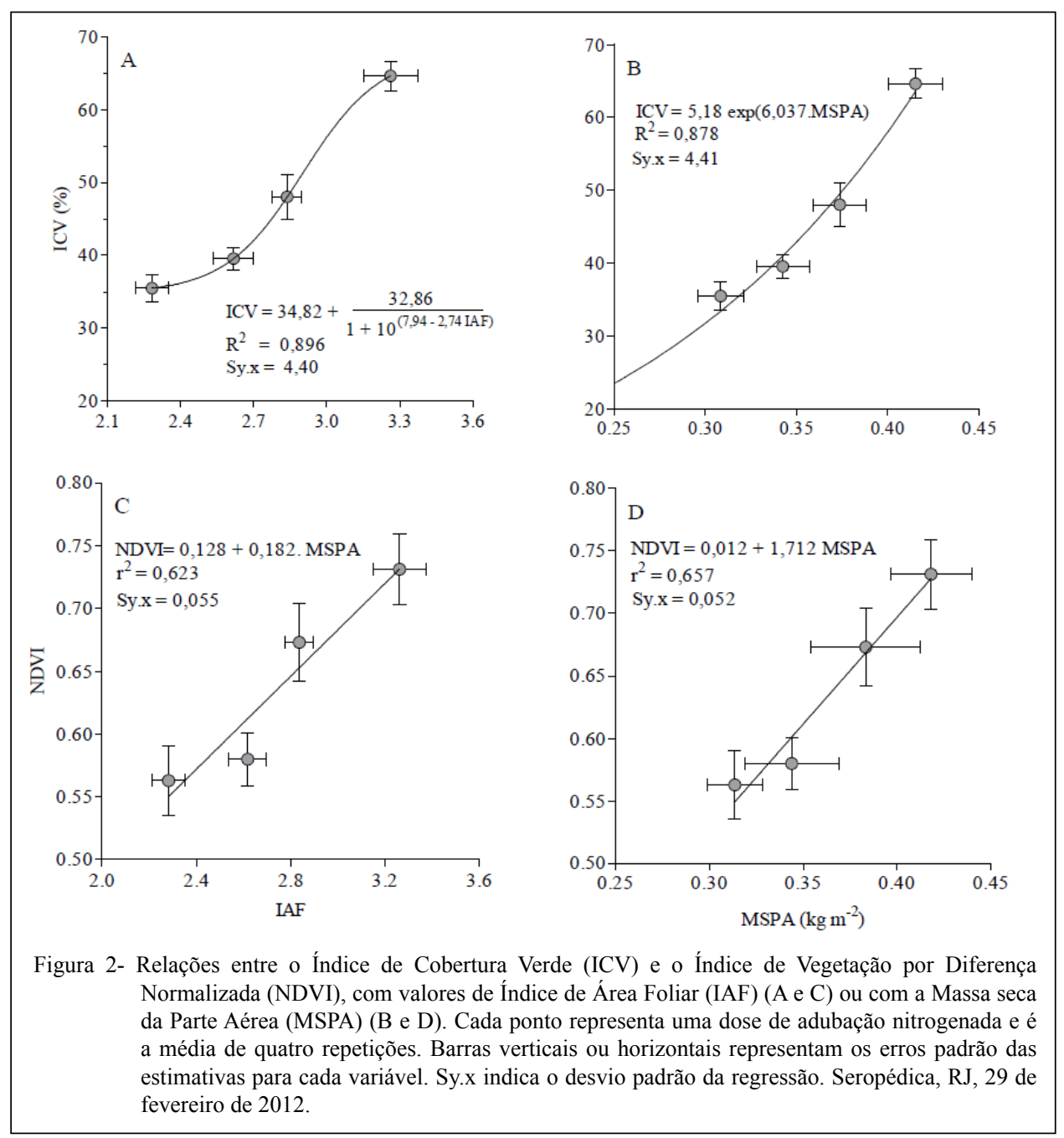

Ciência Rural, v.45, n.4, abr, 2015. 
foliar ou biomassa tomaram por base valores obtidos de forma destrutiva. Entretanto, subsiste o fato de que, com a utilização da informação proveniente da banda do infravermelho próximo, as relações entre os atributos do dossel mostraram um comportamento linear, diferentemente das obtidas com a CD, na banda visível do espectro solar.

Não foram observadas as tendências de saturação relatadas para outras espécies vegetais com o uso do NDVI (OLLINGER, 2011). Então, uma vantagem associada à determinação "in situ" do NDVI seria a atenuação das tendências de resposta não lineares observadas com o uso de câmeras digitais comuns ou com outros métodos manuais de quantificação da cobertura verde, como a frequência de ocorrência (OLIVEIRA et al., 2010). Isto pode ser uma propriedade interessante, pensando na possibilidade de uso do NDVI como um estimador da produção de matéria seca do capim, frente às diferentes doses de adubação nitrogenada.

SCHUT \& KETELAARS (2003) observaram, em mini-dosséis de Lolium perenne, que um índice obtido a partir de imagens classificadas da cobertura vegetal foi um estimador da interceptação luminosa melhor do que o próprio IAF. Esse resultado sugere que os índices de vegetação poderiam ser utilizados como indicadores do IAF, dependendo da finalidade com o qual este último é determinado. Em manejo de pastagens, o conceito de IAF "crítico" corresponde à condição em que o dossel intercepta $95 \%$ da luz incidente (BROUGHAM, 1958), estando associado à maximização da taxa de acúmulo líquido de forragem, e, portanto, ao momento mais adequado para a interrupção da rebrota, por corte ou pastejo. Seria interessante, portanto, comparar o desempenho do ICV e do NDVI com os dos analisadores ópticos do dossel, em valores de IAF mais próximos do crítico, que, para o 'Tifton 85', poderia se situar entre 4,0 (OLIVEIRA et al., 2010) e 4,9 (OLIVEIRA et al., 2000), dependendo da duração do período de rebrota. XIONG et al. (2007) comparou a efetividade de quatro índices ópticos e concluiu que o NDVI foi o melhor indicador dos efeitos da irrigação, sazonalidade e adubação nitrogenada sobre a qualidade de gramados de Cynodon dactylon L. Esse levantamento das potencialidades do NDVI em relação às gramas bermuda suporta os resultados indicados nas figuras $1 \mathrm{C}, 2 \mathrm{C}$ e $2 \mathrm{D}$, se bem que, por serem produtos de uma única data de amostragem, devam ser interpretados com cautela.

\section{CONCLUSÃO}

É possível avaliar a adubação nitrogenada em pastagens de Cynodon spp., utilizando análise de imagens dos dosséis. Tanto o NDVI quanto o ICV podem ser utilizados como indicadores de produtividade do capim 'Tifton 85 '.

\section{REFERÊNCIAS}

ABRAHÃO, S.A. et al. Determination of nitrogen and chlorophyll levels in bean-plant leaves by using spectral vegetation bands and indices. Revista Ciência Agronômica, v.44, p.464-473, 2013. Disponível em: <http://www.scielo.br/scielo.php?pid=S1806$66902013000300007 \&$ script $=$ sci_arttext $>$. Acesso em: 25 fev. 2014. doi: 10.1590/S1806-66902013000300007.

ALDERMAN, P.D. et al. Carbohydrate and nitrogen reserves relative to regrowth dynamics of 'Tifton 85 ' bermudagrass as affected by nitrogen fertilization. Crop Science, v.51, p.17271738, 2011. Disponível em: <https:/www.crops.org/publications/ cs/abstracts/51/4/1727>. Acesso em: 12 dez. 2013. doi: 10.2135/ cropsci2010.09.0516.

BROUGHAM, R.W. Interception of light by the foliage of pure and mixed stands of pasture plants. Australian Journal of Agricultural Research, v.9, p.39-52, 1958. Disponível em: <http://www.scielo.br/ scielo.php?script=sci_arttext\&pid=S0100-204X2001000100023>. Acesso em: 21 jan. 2014. doi: 10.1071/AR9580039.

EMPRESA BRASILEIRA DE PESQUISA AGROPECUÁRIA (EMBRAPA). Sistema Brasileiro de Classificação de Solos. 2.ed. Rio de Janeiro: EMBRAPA/CNPS, 2006. 306p. Disponível em: $\quad<$ http://www.agrolink.com.br/downloads/sistema-brasileirode-classificacao-dos-solos2006.pdf>. Acesso em: 27 de nov. 2013.

JOHNSON, R.A.; WICHERN, D.W. Applied multivariate statistical analysis. 4 ed. Upper Saddle River, New Jersey: PrenticeHall, 1999. 815p. Disponível em: <http://esminfo.prenhall.com/ math/johnsonwichern/data.html >. Acesso em: 2 set. 2013.

LI , Y. et al. Estimating the nitrogen status of crops using a digital camera. Field Crops Research, v.118, p.221-227, 2010. Disponível em: <http:/www.sciencedirect.com/science/article/ pii/S0378429010001358>. Acesso em: 3 jan. 2014. doi: 10.1007/ s13593-011-0041-1.

MANNETJE, L.'t. Measuring biomass of grassland vegetation. In: MANNETJE, L.'t e JONES, R.M. (Eds.). Field and laboratory methods for grassland and animal production research. Wallingford: CABI Publishing, 2000. p.151-177. Disponível em: <http://www.scielo.br/ scielo.php? script $=$ sci_nlinks\&ref $=000101 \&$ pid $=$ S0 100 204X20030008000110002 $8 \& \operatorname{lng}=\mathrm{es}>$. Acesso em: 2 set. 2013. doi: $10.1079 / 9780851993515.0000$.

MATTOS, C.C.L.V. et al. Boletim Agrometeorológico UFRRJ 1996. Floresta e Ambiente, v.5, p.208-215, 1998. Disponível em: $<$ http://www.geocities.ws/floramrural/0208.pdf $>$. Acesso em: 5 set. 2013. doi: 10.4322/floram.2011.058.

MATHWORKS. Image Processing Toolbox for use with MATLAB Release 12. Image Processing Toolbox User's Guide Revised for Version 2.2.2, Massachusetts, 2000. 511p. 
OLIVEIRA, M.A. et al. Análise de crescimento do capim bermuda "Tifton 85" (Cynodon spp.). Revista Brasileira de Zootecnia, v.29, p.1930-1938, 2000. Disponível em: <http:// www.revistasbz.org.br/scripts/revista/sbz1/Artigos/2825.pdf>. Acesso em: 3 nov. 2013.

OLIVEIRA, A.P.P. et al. Respostas do capim-Tifton 85 à aplicação de nitrogênio: cobertura do solo, índice de área foliar e interceptação da radiação solar. Arquivo Brasileiro de Medicina Veterinária e Zootecnia, v.62, p.429-438, 2010. Disponível em: $<$ http://www.scielo.br/scielo.php?script=sci arttext\&pid=S0102>. Acesso em: 9 out. 2013. doi: $10.1590 / 09352010000200024$.

OLLINGER, S.V. Sources of variability in canopy reflectance and the convergent properties of plants. New Phytologist, v.189, p.375-394, 2011. Disponível em: <http://onlinelibrary.wiley.com/ doi/10.1111/j.1469-8137.2010.03536.x/abstract>. Acesso em: 28 jan. 2014. doi: 10.1111/j.1469-8137.2010.03536.x.

QUARESMA, J.P. S. et al. Produção e composição bromatológica do capim-tifton 85 (Cynodon spp.) submetido a doses de nitrogênio. Acta Scientiarum: Animal Sciences, v.33, p.45150, 2011. Disponível em: <http://www.alice.cnptia.embrapa. br/handle/doc/901620>. Acesso em: 16 out. 2013. doi: 10.4025/ actascianimsci.v33i2.9261.

ROSSIELLO, R.O.P.; ANTUNES, M.A.H. Solar radiation utilization by tropical forage grasses: light interception and use efficiency. In: BABATUNDE, E.B. (Ed.) Solar radiation. Rijeka: In Tech, 2012. p.221-244. Disponível em: $<$ http://www.intechopen. com/books/solar-radiation/solar-radiation-utilization-by-tropicalforage-grasses-light-interception-and-use-efficienc $>$. Acesso em: 7 fev. 2013. doi: $10.5772 / 34321$.

ROTZ, J. D. et al. Classification of digital photography for measuring productive ground cover. Rangeland Ecology \& Management, v.61, p.245-248, 2008. Disponível em: <http:// www.researchgate.net/publication/225076678_Classification_of digital_photography_for_measuring_productive_ground_cover>. Acesso em: 24 set. 2014. doi: 10.2111/07-011.1.
SCHUT, A.G.T.; KETELAARS, J.J.M.H. Monitoring grass swards using imaging spectroscopy. Grass and Forage Science, v.58, p.276-286, 2003. Disponível em: <http://www.researchgate. net/publication/229924945_Monitoring_grass_swards sing_imaging_spectroscopy>. Acesso em: 29 out. 2013. doi: 10.1046/j.1365-2494.2003.00379.x.

SENA JÚNIOR, D.G. et al. Discriminação entre estágios nutricionais na cultura de trigo com técnicas de visão artificial e medidor portátil de clorofila. Engenharia Agrícola, v.28, p.187-195, 2008. Disponível em: <http://www.scielo.br/scielo. php? script $=$ sci_arttext\&pid $=\mathrm{S} 0100-69162008000100019>$. Acesso em: 5 dez. 2013. doi: 10.1590/69162008000100019.

SILVA, R.V.M.M. et al. Uso de clorofilometro na avaliação da adubação nitrogenada e potássica no capim Tifton 85 . Revista Brasileira de Saúde e Produção Animal, v.12, p.828-839, 2011. Disponível em: <http://www.scielo.br/pdf/cr/2012nahead/ a35512cr4895.pdf > . Acesso em: 21 jan. 2014. doi: 10.1590/S010384782012005000109 .

VILELA, D. et al. (Eds) Cynodon, forrageiras que estão revolucionando a pecuária brasileira. Juiz de Fora: Embrapa Gado de Leite, 2005. 250p. Disponível em: <http://www.bdpa. cnptia.embrapa.br/busca?b=ad\&id $=594835 \&$ biblioteca $=$ CNPA B\&busca $=$ autoria: $\% 22 \% 22 \& q$ Facets $=$ autoria: $\% 22 \% 22 \&$ sort $=\&$ paginacao $=$ t\&paginaAtual $=120>$. Acesso em: 22 nov. 2013. doi: 10.1590/S0103-84782012005000149

XIONG, X. et al. Bermudagrass seasonal responses to nitrogen fertilization and irrigation detected using optical sensing. Crop Science, v.47, p.1603-1610, 2007. Disponível em: <http://agris. fao.org/agris-search/search.do?recordID=US201300831667> Acesso em: 17 nov. 2013. doi: 10.2135/cropsci2006.06.0400.

ZHAO, D. et al. Assessment of forage biomass and quality parameters of bermudagrass using proximal sensing of pasture canopy reflectance. Grassland Science, v.53, p.39-49, 2007. Disponível em: <http://onlinelibrary.wiley.com/doi/10.1111/ j.1744-697X.2007.00072.x/abstract>. Acesso em: 21 jan. 2014 doi: 10.1111/j.1744-697X.2007.00072.x. 